Elect. Comm. in Probab. 1 (1996) 49-64

\title{
SIMULATIONS AND CONJECTURES FOR DISCONNECTION EXPONENTS
}

\section{EMILY E. PUCKETTE}

Mathematics Department, Occidental College, 1600 Campus Road, Los Angeles, CA 90041, $U S A$

e-mail: eep@oxy.edu

WENDELIN WERNER

Laboratoire de Mathématiques, E.N.S., 45 rue d'Ulm, F-75230 PARIS cedex 05, France e-mail: wwerner@dmi.ens.fr

AMS 1991 Subject classification: 60K35

Keywords and phrases: Brownian Motion, Disconnection Exponents.

\section{Abstract}

Using Monte-Carlo simulations, we estimate numerically disconnection exponents for planar Brownian motions. These simulations tend to confirm conjectures by Duplantier and Mandelbrot.

\section{Introduction}

In recent years, non-intersection exponents for two-dimensional random walks have received a lot of attention. These critical exponents measure the asymptotic probability that the paths of random walks (or Brownian motions) in two dimensions do not intersect up to a large time. One of the tantalizing features of these exponents is that they have been conjectured (see [7], [6]) to take rational values, some of which can be found in Kac's table of unitary highest-weight representation of the Virasoro algebra (see, e.g., Chapter 4 in [8] for a compilation of papers on this subject). For instance, Duplantier and Kwon [6] have conjectured that the critical exponents $\xi_{k}$ (for $k \geq 2$ ) measuring the asymptotics of the probability that $k$ planar random walks starting from distinct points avoid intersection are

$$
\xi_{k}=\frac{4 k^{2}-1}{12}
$$

(or half of these values, depending on the convention.) These conjectures have been confirmed by simulations (only) for $k=2,3$ (see Li-Sokal [16], Duplantier-Kwon [6] and Burdzy-LawlerPolaski [3]).

Similarly, one can define disconnection exponents for two-dimensional paths. These exponents $\eta_{n}$ characterize the asymptotic behavior of the probability that the union of $n$ independent random walks (or Brownian motions) does not disconnect some fixed point from infinity. In other words, this union contains no closed loop around the fixed point. 
Note that the value $\xi_{1}=1 / 4$ for $k=1$ in the formula (1) does not correspond to a nonintersection exponent. In an attempt to interpret this non-trivial value, Duplantier [7] has conjectured that the disconnection exponent $\eta_{1}$ for one path is in fact equal to $\eta_{1}=1 / 4$. If true, this would support the general conjecture (1). One of the aims of the present paper is to see whether simulations rule out or tend to confirm this conjecture for $\eta_{1}$ (we will see that the latter is the case).

Lawler [11] has recently shown that the disconnection exponent for two Brownian motions $\eta_{2}$ is closely related to the Hausdorff dimension $h$ of the "frontier of planar Brownian motion" (also called "self-avoiding Brownian motion" by Mandelbrot [17]; this is the boundary of the unbounded connected component of the complement of the planar Brownian path on a finite time-interval) and that

$$
h=2-\eta_{2} .
$$

Mandelbrot [17] has conjectured by analogy with conjectures on self-avoiding random walks that $h=4 / 3$, which would imply that $\eta_{2}=2 / 3$. We will also see that simulations support this conjecture. See also [4], [10] for other applications and features of the connection between critical exponents and fine properties of the planar Brownian path.

We will also give some new conjectures on disconnection exponents and non-intersection exponents between packs of Brownian motions. These conjectures formally link the number of Brownian motions allowed to intersect each other (the number of Brownian motions in each pack) with the central charge of the considered Virasoro algebra.

The paper is organized as follows. We first concentrate on the disconnection exponent for one random walk. We recall some relevant facts on $\eta_{1}$ in section 2 , and we present some simulations and Monte-Carlo estimates in section 3. In section 4, we focus on $\eta_{2}$ and then give a brief statement of results from simulations for three random walks. Finally, in section 5, we give some further conjectures.

\section{Notation}

We will often identify $\mathbb{R}^{2}$ and the complex plane $\mathbb{C}$. The real and imaginary parts of a complex number $z$ will be denoted $\Re(z)$ and $\Im(z)$ respectively. For all $r>0, C_{r}$ will denote the circle $\{z \in \mathbb{C}:|z|=r\}$. We will say that a compact set $K \subset \mathbb{C}$ disconnects a point $a \in \mathbb{C}$ from infinity if and only if it contains a closed loop around $a$.

$S$ will denote the simple random walk on the lattice $\mathbb{Z}^{2}$ starting from 0 . We also define the associated continuous function $\hat{S}$ as follows: $\hat{S}(n)=S(n)$ for all non-negative integer $n$, and for all $t \in[0,1]$, we put

$$
\hat{S}(n+t)=t S(n+1)+(1-t) S(n) .
$$

We will say that $S[0, n]$, the path of the random walk from time 0 to time $n$, disconnects $a \in \mathbb{C}$ from infinity if $\hat{S}[0, n]=\cup_{j=0}^{n-1}[S(j), S(j+1)]$ disconnects $a$ from infinity.

\section{The Disconnection Exponent for One Walk}

Let us first recall the definition of $\eta_{1}$ and some rigorous results. Let $B=\left\{B_{t}: t \geq 0\right\}$ denote a complex Brownian motion started from 1 under the probability measure $\mathbb{P}$ and let

$$
T_{r}=\inf \left\{t>0: B_{t} \in C_{r}\right\}
$$


for $r>0$. Then $\eta_{1}$ describes the asymptotics of the probability

$$
p_{r}=\mathbb{P}\left\{B\left[0, T_{r}\right] \text { does not disconnect } 0 \text { from infinity }\right\}
$$

when $r \rightarrow \infty$. More precisely, a straightforward subadditivity argument (see, e.g., [9]) shows that the limit

$$
\eta_{1}=\lim _{r \rightarrow \infty}-\frac{\ln p_{r}}{\ln r}
$$

exists and is finite and strictly positive. Lawler [13] has recently shown that in fact the following stronger result holds for some constant $c \in(0, \infty)$ :

$$
\lim _{r \rightarrow \infty} r^{\eta_{1}} p_{r}=c .
$$

The best current rigorous bounds are

$$
\frac{1}{2 \pi} \leq \eta_{1}<.469
$$

See Werner [19], [18] and Burdzy-Lawler [2]. Lawler and Puckette [15] have shown that the analogous exponent for the nearest neighbor random walk in $\mathbb{Z}^{2}$ exists and is in fact also equal to $\eta_{1}$. This allows us to confidently identify the estimated exponent obtained via random walk simulations with an approximation of $\eta_{1}$. Furthermore, from well-known estimates such as

$$
\mathbb{P}\left\{T_{r}>r^{2+\epsilon} \text { or } T_{r}<r^{2-\epsilon}\right\} \leq c e^{-a r}
$$

for all $r>1$ and for some fixed $a, c>0$, one can show that the probabilities

$$
p_{t}^{\prime}=\mathbb{P}\{B[0, t] \text { does not disconnect } 0 \text { from infinity }\}
$$

and

$$
q_{n}^{\prime}=\mathbb{P}\{S[0, n] \text { does not disconnect } 0 \text { from infinity }\}
$$

satisfy

$$
\lim _{t \rightarrow \infty}-\frac{\ln p_{t}^{\prime}}{\ln t}=\lim _{n \rightarrow \infty}-\frac{\ln q_{n}^{\prime}}{\ln n}=\frac{\eta_{1}}{2}
$$

(see [9], [15]). The factor $1 / 2$ has lead to various definitions of these disconnection exponents (with, for instance, $\eta_{1} / 2$ instead of $\eta_{1}$ ). Note that (3) implies that

$$
q_{n}^{\prime}=L(n) n^{-\eta_{1} / 2},
$$

where $\lim _{n \rightarrow \infty} \ln L(n) / \ln n=0$. The methods developed in Lawler [14] suggest that

$$
0<\liminf _{n \rightarrow \infty} L(n) \leq \limsup _{n \rightarrow \infty} L(n)<\infty .
$$

\section{Monte-Carlo Simulations for $\eta_{1}$}

We considered the events

$$
E_{n}=\{S[0, n] \text { disconnects }(1 / 2,1 / 2) \text { from infinity }\} .
$$


Let $D$ denote the smallest integer for which $E_{D}$ holds. It is not hard to see that

$$
\lim _{n \rightarrow \infty} \frac{\ln \mathbb{P}\{D>n\}}{\ln q_{n}^{\prime}}=1,
$$

and so our consideration of $D$ will produce estimates of $\eta_{1}$ via equation (3).

Let $N_{n}$ denote the "algebraic winding number" of $S$ around $a=(1 / 2,1 / 2)$ defined as follows: $N_{0}=0$ and for all $n \geq 0$,

- $N_{n+1}=N_{n}-1$ if $\Re\left(S_{n}\right) \leq 0, \Im\left(S_{n}\right)=0$ and $\Im\left(S_{n+1}\right)=1$,

- $N_{n+1}=N_{n}+1$ if $\Re\left(S_{n}\right) \leq 0, \Im\left(S_{n}\right)=1$ and $\Im\left(S_{n+1}\right)=0$,

- $N_{n+1}=N_{n}$ otherwise.

In other words, $N_{n+1}=N_{n}-1$ or $N_{n+1}=N_{n}+1$ if $S$ jumps up or down, respectively, across the half-line $\{(x, 1 / 2): x \in(-\infty, 1 / 2)\}$ between the times $n$ and $n+1$. We note that:

\section{Lemma 1}

$$
D=\inf \left\{n>0: \exists p<n \text { where } S_{n}=S_{p} \text { and } N_{n} \neq N_{p}\right\}
$$

Proof Put

$$
D^{\prime}=\inf \left\{n>0: \exists p<n \text { where } S_{n}=S_{p} \text { and } N_{n} \neq N_{p}\right\} .
$$

It is clear that $D^{\prime} \geq D$, because if $p<n, N_{p} \neq N_{n}$ and $S_{n}=S_{p}$, then $\left(\hat{S}_{u}, u \in[p, n]\right)$ is a closed loop, and the index of that loop around $a$ is $N_{n}-N_{p} \neq 0$.

Now, suppose that $D^{\prime}>D$. In other words, for all $(s, t) \in[0, D]^{2}$ such that $\hat{S}_{s}=\hat{S}_{t}$, one has

$$
\int_{s}^{t} \frac{d \hat{S}_{u}}{\hat{S}_{u}-a}=0
$$

But as $S[0, D]$ contains a closed loop around $a$, it is easy to see that for some finite family $\left(s_{0}, t_{0}, s_{1}, t_{1}, \ldots, s_{k}, t_{k}\right) \in[0, D]^{2 k+2}$, such that $S_{t_{0}}=S_{s_{1}}, S_{t_{1}}=S_{s_{2}}, \ldots, S_{t_{k-1}}=S_{s_{k}}$ and $S_{t_{k}}=S_{s_{0}}$, one has

$$
\sum_{j=0}^{k} \int_{s_{j}}^{t_{j}} \frac{d \hat{S}_{u}}{\hat{S}_{u}-a} \neq 0
$$

But (5) implies that, for all $j \in\{0, \ldots, k-1\}$,

$$
\int_{t_{j}}^{s_{j+1}} \frac{d \hat{S}_{u}}{\hat{S}_{u}-a}=0
$$

and that $\int_{s_{0}}^{t_{k}} d \hat{S}_{u} /\left(\hat{S}_{u}-a\right)=0$. Combined with (6), this implies that

$$
0=\int_{s_{0}}^{t_{k}} \frac{d \hat{S}_{u}}{\hat{S}_{u}-a}=\sum_{j=0}^{k} \int_{s_{j}}^{t_{j}} \frac{d \hat{S}_{u}}{\hat{S}_{u}-a}+\sum_{j=0}^{k-1} \int_{t_{j}}^{s_{j+1}} \frac{d \hat{S}_{u}}{\hat{S}_{u}-a} \neq 0 .
$$

Hence, $D^{\prime} \leq D$. 
Using this remark, it is easy for a computer to work out the value of $D$ from a simulation of $S$. With an elementary C-program, we can obtain an approximation of $\eta_{1}$ via methods used for the non-intersection exponent simulations (see [3], [6] and [16]). Our aim here is not to derive high-precision estimates but rather to see whether the conjecture $\eta_{1}=1 / 4$ is ruled out by simulations or not.

Let us fix $m<M$. Suppose we simulate $W$ random walks. Define $A(n)$ to be the number of random walks (out of $W$ ) which do not disconnect $a$ before the $n$-th step. The empirical exponent $\tilde{\eta}_{1}(m, M)$ is defined as

$$
\tilde{\eta}_{1}(m, M)=\frac{2 \ln (A(m) / A(M))}{\ln (M / m)} .
$$

Note that we consider $m<M$ (just as in [3], [6] and [16]) in order to avoid any difficulties that, for example, the values of $L(n)$ for large $n$ 's (see (4)) might cause.

When $W \rightarrow \infty, \tilde{\eta}_{1}(m, M)$ converges almost surely towards

$$
\eta_{1}(m, M)=\frac{2 \ln \left(q_{m}^{\prime} / q_{M}^{\prime}\right)}{\ln (M / m)} .
$$

Of course, when $M \rightarrow \infty, m \rightarrow \infty$ and $m \ll M, \eta_{1}(m, M)$ converges towards $\eta_{1}$.

We simulated $W=9 \times 10^{6}$ walks of length 50000, which produced the following values of $A(n)$ which are plotted in the graph below.

\begin{tabular}{|r|r|r|r|r|r|}
\hline $\mathrm{n}$ & 5000 & 10000 & 15000 & 20000 & 25000 \\
\hline $\mathrm{A}(\mathrm{n})$ & 4722973 & 4345105 & 4136541 & 3993170 & 3885387 \\
\hline
\end{tabular}

\begin{tabular}{|r|r|r|r|r|r|}
\hline $\mathrm{n}$ & 30000 & 35000 & 40000 & 45000 & 50000 \\
\hline $\mathrm{A}(\mathrm{n})$ & 3799862 & 3729841 & 3670014 & 3617394 & 3570793 \\
\hline
\end{tabular}

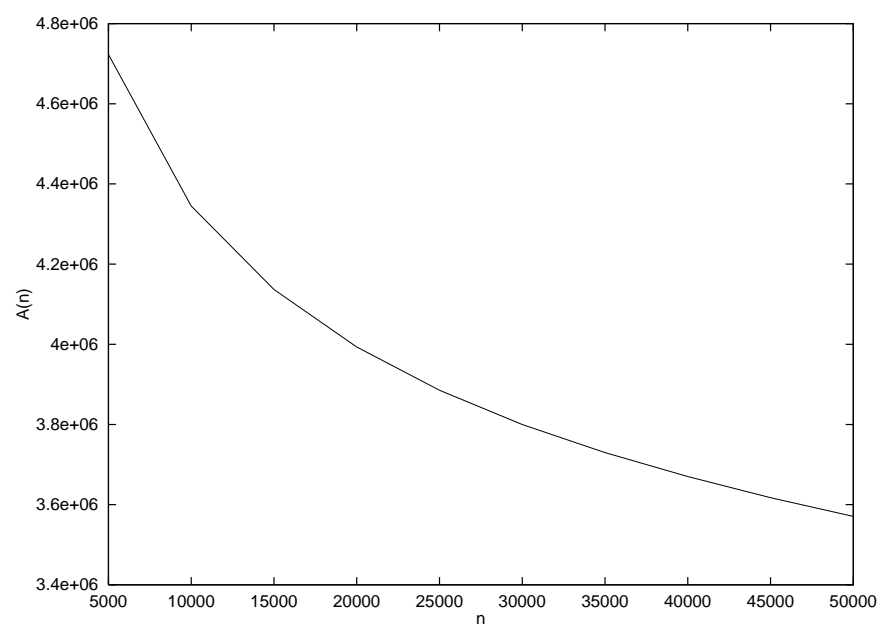


From these values of $A(n)$, we calculate values of $\tilde{\eta}_{1}(m, M)$. We also determine confidence intervals for our estimates of $\eta_{1}$. To do this, we define $p=p(m, M)=p_{M}^{\prime} / p_{m}^{\prime}$ and let $\bar{p}=A(M) / A(m)$ be our estimator of $p$. Then an approximate $95 \%$ confidence interval for $p$ would be given by $\left[\bar{p}_{-}, \bar{p}_{+}\right]$where

$$
\bar{p}_{ \pm}=\bar{p} \pm 2\left[\frac{\bar{p}(1-\bar{p})}{A(m)}\right]^{1 / 2}
$$

This in turn produces an approximate $95 \%$ confidence interval for $\eta_{1}(m, M)$ with bounds given by

$$
\tilde{\eta}_{1}^{ \pm}=\tilde{\eta}_{1}^{ \pm}(m, M)=2 \frac{\log \left(\bar{p}_{\mp}\right)}{\log (m / M)} .
$$

We now present the results obtained for $M=50000$ with a plot of the three functions $\tilde{\eta}_{1}^{-}(m, 50000), \tilde{\eta}_{1}(m, 50000)$ and $\tilde{\eta}_{1}^{+}(m, 50000)$.

\begin{tabular}{|l|c|c|}
\hline$m \times 10^{-3}$ & $\tilde{\eta}_{1}(m, 50000)$ & Confidence interval \\
\hline 5 & .242902 & {$[.242448, .243356]$} \\
\hline 10 & .243889 & {$[.243334, .244444]$} \\
\hline 15 & .244312 & {$[.243661, .244962]$} \\
\hline 20 & .244022 & {$[.243271, .244774]$} \\
\hline 25 & .243628 & {$[.242759, .244497]$} \\
\hline 30 & .243437 & {$[.242420, .244455]$} \\
\hline 35 & .244356 & {$[.243131, .245582]$} \\
\hline 40 & .245717 & {$[.244158, .247278]$} \\
\hline 45 & .246130 & {$[.243850, .248410]$} \\
\hline
\end{tabular}

Table of values for $\tilde{\eta}_{1}(m, 50000)$ 


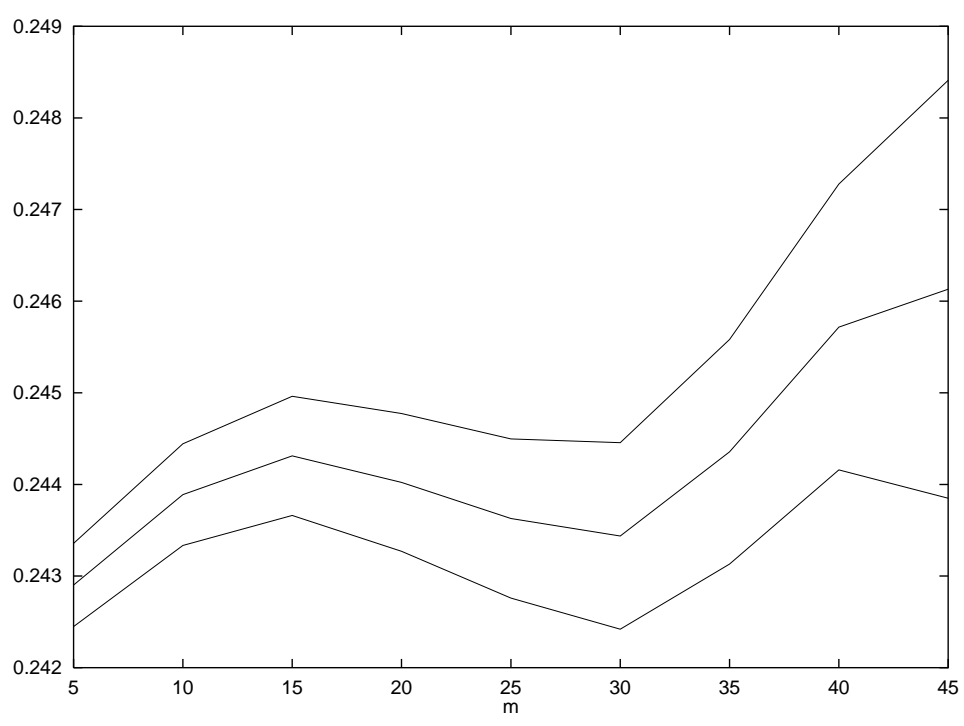

Plot of $\tilde{\eta}_{1}(m, 50000)$ with confidence intervals

The estimated values for $\eta_{1}(m, M)$ where $M \neq 50000$ are presented in the appendix. Here is a three-dimensional plot of this function:

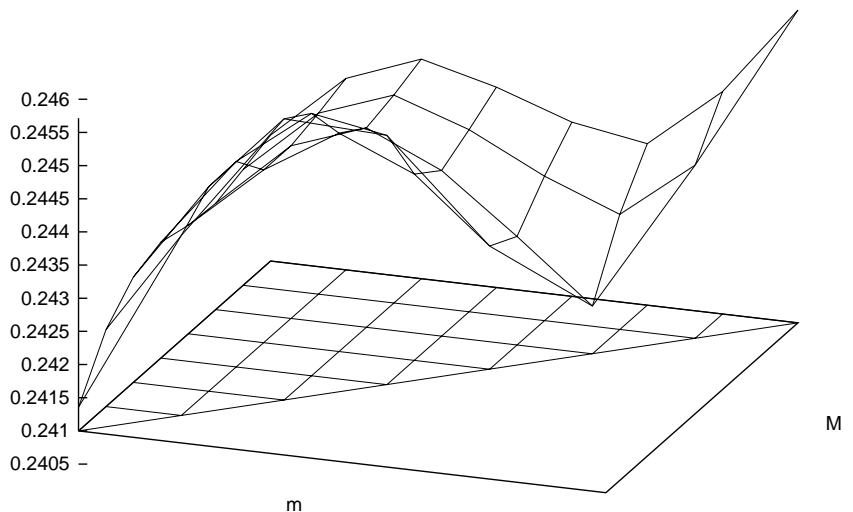

It is striking that $\eta_{1}(m, M)$ is roughly increasing in $m$ and $M$ (see also Appendix 1 ). It is therefore not surprising that the simulations yield slightly smaller estimates than the conjectured value. Just as for non-intersection exponents, it is probably just a consequence of the fact that

$$
f_{1}(r)=-\log p_{r} / \log r
$$

is increasing in $r$. Note also that the big oscillations near the diagonal (when $M$ and $m$ are close) correspond to a domain where the confidence intervals are larger. 


\section{The Disconnection Exponent for More Than One Walk}

One can define disconnection exponents for several Brownian motions in a very similar way. Let $n \geq 2$, and consider $n$ independent planar Brownian motions $B^{1}, \ldots, B^{n}$ starting from $x_{1}, \ldots, x_{n}$ under the probability measure $\mathbb{P}_{X}$ (where $\left.X=\left(x_{1}, \ldots, x_{n}\right) \in \mathbb{C}^{n}\right)$. For all $r>0$ and $i=1, \ldots, n$, we define

$$
T_{r}^{i}=\inf \left\{t>0: B_{t}^{i} \in C_{r}\right\} .
$$

We consider the probability

$$
p_{r}^{n}=\sup _{X \in\left(C_{1}\right)^{n}} \mathbb{P}_{X}\left\{\bigcup_{1 \leq j \leq n} B^{j}\left[0, T_{r}^{j}\right] \text { does not disconnect } 0 \text { from infinity }\right\} .
$$

Arguments similar to those above (see, e.g., [18]) show that

$$
\eta_{n}=\lim _{r \rightarrow \infty}-\frac{\ln p_{r}^{n}}{\ln r}
$$

exists and is finite and strictly positive. See again [2], [18] for rigorous bounds. There is (up to now) no proof of the fact that the same exponent for simple random walks exists and is equal to $\eta_{n}$. It is however believed to be true. The methods used in [1] or in [15] to show equivalence between other exponents do not seem to generalize smoothly.

We focus on $\eta_{2}$ because of the connection (2) with the Hausdorff dimension of the 'frontier' of planar Brownian motion derived by Lawler [12]. Consider two independent random walks $S^{1}$ and $S^{2}$ in $Z^{2}$ starting from 0 . We define the winding numbers $N^{1}$ and $N^{2}$ of $S^{1}$ and $S^{2}$, respectively, just as $N$ was defined in section 3. Again, if

$$
E_{n}=\left\{S^{1}[0, n] \cup S^{2}[0, n] \text { disconnects }(1 / 2,1 / 2) \text { from infinity }\right\},
$$

and if $D$ denotes the first disconnecting time

$$
D=\inf \left\{n>0: E_{n} \text { holds }\right\},
$$

then (just as in the previous section)

$$
D=\inf \left\{n>0: \exists p \leq n, \exists(i, j) \in\{1,2\}^{2} \text { such that } S_{n}^{i}=S_{p}^{j} \text { and } N_{n}^{i} \neq N_{p}^{j}\right\} .
$$

So, it is again easy to obtain $D$ from simulations of $S^{1}$ and $S^{2}$.

We simulated $W=1.5 \times 10^{6}$ pairs of walks of length 75000 . Let $A(n)$ represent the number of pairs of walks (out of $W$ pairs) that have not disconnected $a=(1 / 2,1 / 2)$ after $n$ steps.

\begin{tabular}{|r|r|r|r|r|r|}
\hline $\mathrm{n}$ & 7500 & 15000 & 22500 & 30000 & 37500 \\
\hline $\mathrm{A}(\mathrm{n})$ & 199569 & 158980 & 139230 & 126505 & 117438 \\
\hline $\mathrm{r}$ & 45000 & 52500 & 60000 & 67500 & 75000 \\
\hline $\mathrm{A}(\mathrm{n})$ & 110627 & 105139 & 100508 & 96737 & 93368 \\
\hline
\end{tabular}




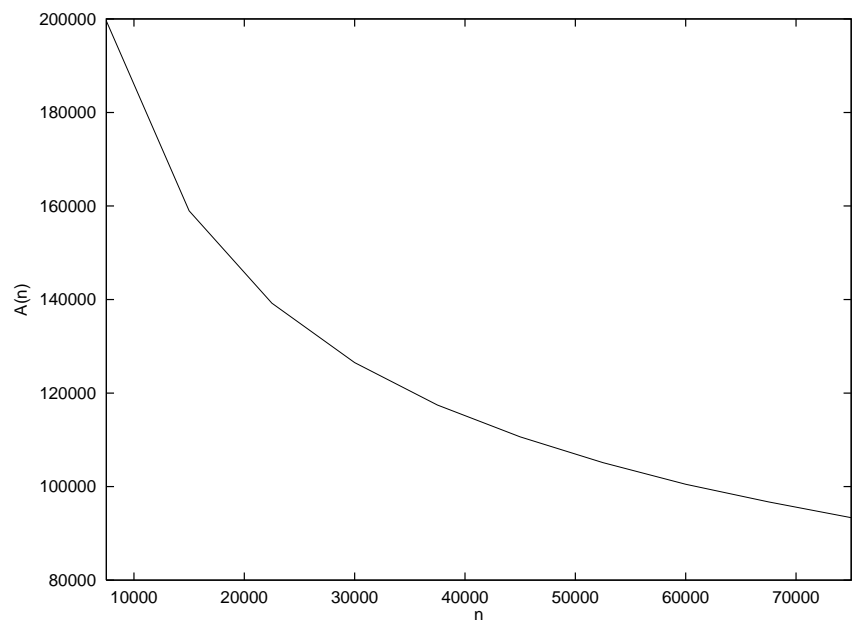

Plot of $A(n)$ for two walks and $W=1500000$.

Considering the empirical probabilities of no disconnection $\tilde{p}_{n}=A(n) / W$ up to step $n$, we compute the apparent exponent

$$
\tilde{\eta}_{2}(m, M)=\frac{2 \log \left(\tilde{p}_{m} / \tilde{p}_{M}\right)}{\log (M / m)}
$$

for $m<M$. We obtain the following values and $95 \%$ confidence intervals for $\tilde{\eta}_{2}(m, 75000)$ :

\begin{tabular}{|l|c|c|}
\hline$m \times 10^{-3}$ & $\tilde{\eta}_{2}(m, 75000)$ & Confidence interval \\
\hline 7.5 & .659790 & {$[.655653, .663947]$} \\
\hline 15 & .661386 & {$[.656172, .666622]$} \\
\hline 22.5 & .663767 & {$[.657538, .670019]$} \\
\hline 30 & .662962 & {$[.655663, .670287]$} \\
\hline 37.5 & .661798 & {$[.653261, .670361]$} \\
\hline 45 & .664084 & {$[.653975, .674219]$} \\
\hline 52.5 & .665786 & {$[.653519, .678080]$} \\
\hline 60 & .660460 & {$[.644837, .676109]$} \\
\hline 67.5 & .672876 & {$[.649704, .696077]$} \\
\hline
\end{tabular}

Table of values for $\tilde{\eta}_{2}(m, 75000)$ 


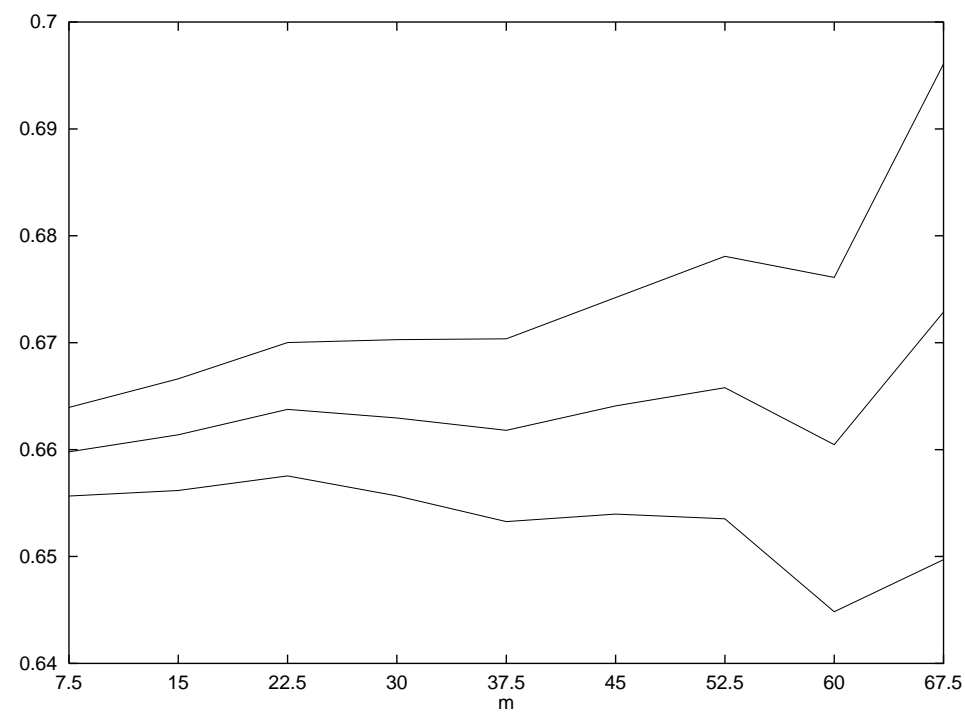

Plot of $\tilde{\eta}_{2}(m, 75000)$ with confidence interval.

The following is a three-dimensional plot of the estimated values for $\eta_{2}(m, M)$ where $M \neq$ 75000 .

Plot of $\tilde{\eta}_{2}(m, M)$.

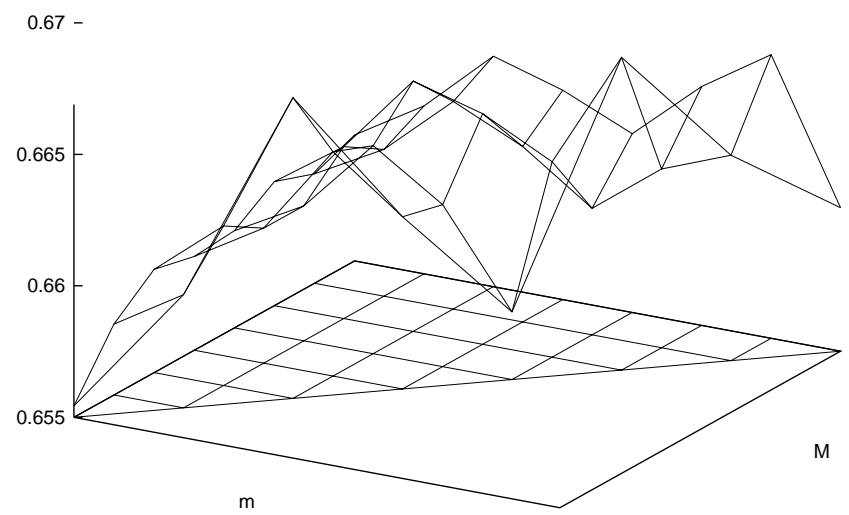

The corresponding values and confidence intervals are listed in Appendix 2. Note again that the big oscillations near the diagonal correspond to a domain where the confidence interval is larger.

In attempting to obtain an estimate for $\eta_{3}$, we simulated $2 \times 10^{6}$ sets of three walks, each of length 30000. Again $A(n)$ represents the number of times no disconnection has occurred before step $n$. The values of $A(n)$ for some values of $n$ are listed below : 


\begin{tabular}{|r|r|r|r|}
\hline$n$ & 6000 & 12000 & 30000 \\
\hline$A(n)$ & 69608 & 47683 & 28929 \\
\hline
\end{tabular}

The values of $A(n)$ are (not surprisingly) small; it is therefore more difficult to obtain good approximations using our primitive method, which we plan to improve. The apparent exponent $\tilde{\eta}_{3}$ for $m=6000$ and $M=30000$ computed as for $\tilde{\eta}_{2}$ is

$$
\tilde{\eta}_{3}=1.091 \pm .014
$$

which is not very informative.

\section{Other exponents and more conjectures}

We did not resist the temptation of making conjectures on the exact value of disconnection exponents. A conjecture, which fits with all simulations and estimates we know of is:

$$
\eta_{n}=\frac{n^{2}}{2(n+1)} .
$$

In particular, $\eta_{1}=1 / 4, \eta_{2}=2 / 3, \eta_{3}=9 / 8$. One also has $\eta_{n}<n / 2$, but $\lim _{n \rightarrow \infty} \eta_{n} / n=1 / 2$ (see [20]).

It is also worthwhile considering the generalizations of the non-intersection exponents $\xi_{k}$, where $n$ Brownian motions start from each of $k$ distinct points, and where one looks at the probability of no intersection between any two Brownian motions starting from different points. We call these exponents, denoted by $\pi(k, n)$, the non-intersection exponents between packs of Brownian motions. Let us define them rigorously: for $1 \leq j \leq k, 1 \leq i \leq n, B^{i, j}$ will denote $n \times k$ independent planar Brownian motions starting from $B_{0}^{i, j}=x_{i, j}$, where $X=\left(x_{i, j}\right)_{1 \leq i \leq n, 1 \leq j \leq k} \in \mathbb{C}^{n \times k}$ under the probability measure $\mathbb{P}_{X}$. We let:

$$
T_{r}^{i, j}=\inf \left\{t>0:\left|B_{t}^{i, j}\right|=r\right\} .
$$

Then for all $r>1$, we consider the events $F_{r}^{k, n}$ defined by

$$
\left\{B^{i, j}\left[0, T_{r}^{i, j}\right] \cap B^{i^{\prime}, j^{\prime}}\left[0, T_{r}^{i^{\prime}, j^{\prime}}\right]=\emptyset \quad \forall i, i^{\prime} \in\{1, \ldots, n\}, \forall j \neq j^{\prime} \in\{1, \ldots, k\}\right\},
$$

and the probabilities:

$$
p_{r}^{k, n}=\sup _{X \in\left(C_{1}\right)^{n \times k}} \mathbb{P}_{X}\left(F_{r}^{k, n}\right) .
$$

Then, it is straightforward to check that for all $r, r^{\prime}>1$,

$$
p_{r r^{\prime}}^{k, n} \leq p_{r}^{k, n} p_{r^{\prime}}^{k, n}
$$

Hence, by subadditivity (see, e.g., Lawler [9]):

$$
\pi(k, n)=\lim _{r \rightarrow \infty}-\frac{\ln p_{r}^{k, n}}{\ln r}
$$

exists and is strictly positive. On the other hand, it is easy to check that $\pi(k, n)<\infty$ by considering, for instance, the probability that all Brownian motions stay in well-chosen cones (see, e.g., [20]). 
Of course, $\pi(k, 1)=\xi_{k}$. By analogy with $\xi_{1}=\eta_{1}$, we can expect that formally, $\pi(1, n)=\eta_{n}$. A reasonable conjecture is

$$
\pi(k, n)=n \frac{((n+1) k)^{2}-1}{2(n+1)(n+2)} .
$$

This conjecture contains all previous conjectures, and if true, would link the central charge of the considered Virasoro algebra with the number of Brownian motions started from each point. Of course, this is tentative and may very well be ruled out by simulations in the near future. We will be testing these conjectures shortly and plan to provide links to the results of these simulations in the list of follow-up papers, or in the comment section that the electronic support makes available. 
Appendix 1: Table of other values for $\tilde{\eta}_{1}$

\begin{tabular}{|c|c|c|c|}
\hline$m \times 10^{-3}$ & $M \times 10^{-3}$ & $\tilde{\eta}_{1}(m, M)$ & Confidence interval \\
\hline 5 & 10 & .240609 & $239826, .241392]$ \\
\hline 5 & 15 & 241356 & {$[.240726, .241987]$} \\
\hline 5 & 20 & 242161 & {$[.241593, .242729]$} \\
\hline 5 & 25 & .242589 & {$[.242058, .243120]$} \\
\hline 5 & 30 & .242749 & {$[.242243, .243255]$} \\
\hline 5 & 35 & .242635 & {$[.242147, .243123]$} \\
\hline 5 & 40 & .242606 & {$[.242132, .243081]$} \\
\hline 5 & 45 & .242747 & {$[.242284, .243210]$} \\
\hline 10 & 15 & .242635 & {$[.241572, .243698]$} \\
\hline 10 & 20 & 243713 & {$[.242891, .244535]$} \\
\hline 10 & 25 & 244087 & {$[.243367, .244808]$} \\
\hline 10 & 30 & .244099 & {$[.243438, .244761]$} \\
\hline 10 & 35 & 243605 & {$[.243134, .244378]$} \\
\hline 10 & 40 & .243605 & {$[.243012, .244199]$} \\
\hline 10 & 45 & .243732 & {$[.243160, .244305]$} \\
\hline 15 & 20 & .245233 & $.243937, .246528]$ \\
\hline 15 & 25 & 245240 & {$[.244261, .246219]$} \\
\hline 15 & 30 & 244956 & {$[.244111, .245800]$} \\
\hline 15 & 35 & 244293 & {$[.243526, .245060]$} \\
\hline 15 & 40 & .244007 & {$[.243292, .244722]$} \\
\hline 15 & 45 & 244137 & {$[.243459, .244815]$} \\
\hline 20 & 25 & .245248 & $.243755, .246743]$ \\
\hline 20 & 30 & 244759 & {$[.243646, .245873]$} \\
\hline 20 & 35 & 243809 & {$[.242859, .244760]$} \\
\hline 20 & 40 & 243498 & {$[.242641, .244355]$} \\
\hline 20 & 45 & 243749 & {$[.242953, .244544]$} \\
\hline 25 & 30 & .242491 & $.244160, .245830]$ \\
\hline 25 & 35 & .242855 & $.241624, .244087]$ \\
\hline 25 & 40 & .242635 & {$[.241590, .243681]$} \\
\hline 25 & 45 & .243179 & $.242240, .244189]$ \\
\hline 30 & 35 & .241311 & $.239487, .243135]$ \\
\hline 30 & 40 & 241720 & $.240379, .243062]$ \\
\hline 30 & 45 & .242738 & $.241601, .243875]$ \\
\hline 35 & 40 & 242192 & $.240212, .244173]$ \\
\hline 35 & 45 & .243613 & $.242160, .245066]$ \\
\hline 40 & 45 & .245224 & {$[.243086, .247362]$} \\
\hline
\end{tabular}


Appendix 2. Table of other values for $\tilde{\eta}_{2}$

\begin{tabular}{|l|l||l|l|}
\hline$m \times 10^{-3}$ & $M \times 10^{-3}$ & $\tilde{\eta}_{2}(m, M)$ & Confidence interval \\
\hline \hline 7.5 & 15 & .656085 & {$[.649565, .662619]$} \\
7.5 & 22.5 & .655432 & {$[.650074, .660805]$} \\
7.5 & 30 & .657693 & {$[.652793, .662610]$} \\
7.5 & 37.5 & .658925 & {$[.654281, .663586]$} \\
7.5 & 45 & .658566 & {$[.654094, .663056]$} \\
7.5 & 52.5 & .658691 & {$[.654339, .663061]$} \\
7.5 & 60 & .659718 & {$[.655453, .664003]$} \\
7.5 & 67.5 & .659163 & {$[.654971, .663374]$} \\
\hline 15 & 22.5 & .654316 & {$[.645006, .663644]$} \\
15 & 30 & .659302 & {$[.651978, .666644]$} \\
15 & 37.5 & .661074 & {$[.654572, .667595]$} \\
15 & 45 & .660132 & {$[.654104, .666178]$} \\
15 & 52.5 & .660133 & {$[.654413, .665874]$} \\
15 & 60 & .661535 & {$[.656026, .667065]$} \\
15 & 67.5 & .660581 & {$[.655242, .665942]$} \\
\hline 22.5 & 30 & .666329 & {$[.654520, .678157]$} \\
22.5 & 37.5 & .666438 & {$[.657408, .675488]$} \\
22.5 & 45 & .663533 & {$[.665680, .671408]$} \\
22.5 & 52.5 & .662917 & {$[.655723, .670132]$} \\
22.5 & 60 & .664519 & {$[.657747, .671314]$} \\
22.5 & 67.5 & .662893 & {$[.656437, .669372]$} \\
\hline 30 & 37.5 & .666578 & {$[.652585, .680593]$} \\
30 & 45 & .661550 & {$[.651053, .672069]$} \\
30 & 52.5 & .661163 & {$[.652115, .670234]$} \\
30 & 60 & .663768 & {$[.655528, .672032]$} \\
30 & 67.5 & .661674 & {$[.653993, .669380]$} \\
\hline 37.5 & 45 & .655395 & {$[.639521, .671292]$} \\
37.5 & 52.5 & .657571 & {$[.645718, .669448]$} \\
37.5 & 60 & .662434 & {$[.652254, .672639]$} \\
37.5 & 67.5 & .659813 & {$[.650639, .669011]$} \\
\hline 45 & 52.5 & .660145 & {$[.642330, .677981]$} \\
45 & 60 & .666895 & {$[.653643, .680172]$} \\
45 & 67.5 & .661799 & {$[.650573, .673051]$} \\
\hline 52.5 & 60 & .674687 & {$[.654870, .694531]$} \\
52.5 & 67.5 & .662814 & {$[.648361, .677293]$} \\
\hline 60 & 67.5 & .649353 & {$[.649704, .696077]$} \\
\hline
\end{tabular}




\section{References}

[1] K. Burdzy, G.F. Lawler (1990): Non-intersection exponents for random walk and Brownian motion. Part I: Existence and an invariance principle. Probab. Theor. Rel. Fields 84, 393-410.

[2] K. Burdzy and G.F. Lawler (1990): Non-intersection exponents for random walk and Brownian motion. Part II: Estimates and applications to a random fractal, Ann. Probab. 18, 981-1009.

[3] K. Burdzy, G.F. Lawler and T. Polaski (1989): On the critical exponent for random walk intersections, J. Stat. Phys. 56, 1-12.

[4] K. Burdzy and W. Werner (1996): No triple point of planar Brownian motion is accessible. Ann. Probab. 24, 125-147.

[5] M. Cranston and T. Mountford (1991): An extension of a result by Burdzy and Lawler, Prob. Th. Relat. Fields 89, 487-502.

[6] B. Duplantier and K.H. Kwon (1988): Conformal invariance and intersection of random walks. Phys. Rev. Lett. 61, 2514-2517.

[7] B. Duplantier, G.F. Lawler, J.-F. Le Gall and T.J. Lyons (1993): The geometry of the Brownian curve, in Probabilités et Analyse Stochastique, Tables Rondes de St-Chéron, Jan. 1992, Bull. Soc. Math. (2) 117, 91-106.

[8] P. Goddard and D. Olive Eds. (1988): Kac-Moody and Virasoro algebras, Adv. Series in Math. Phys. vol. 3, World Scientific

[9] G.F. Lawler (1991): Intersection of Random Walks, Birkhaüser, Boston

[10] G.F. Lawler (1993): A discrete analogue of a theorem of Makarov. Combinatorics, Probability and Computing 2, 181-200.

[11] G.F. Lawler (1995): Haussdorff dimension of cut points for Brownian motion, Electronic J. Probab. 1, paper no.2.

[12] G.F. Lawler (1996): The dimension of the frontier of planar Brownian motion, Electronic Comm. Prob. 1, 29-47.

[13] G.F. Lawler (1995): Nonintersecting planar Brownian motions, Math. Phys. El. J. 1, paper no.4.

[14] G.F. Lawler (1995): Cut times for simple random walk, Duke University preprint 95-04.

[15] G.F. Lawler and E.E. Puckette (1996): The disconnection exponent for simple random walk, Israel J. Math. (to appear)

[16] B. Li, A.D. Sokal (1990): High-precision Monte Carlo test of the conformal-invariance predictions for two-dimensional mutually avoiding walks, J. Stat. Phys 61, 723-748.

[17] B.B. Mandelbrot (1982): The fractal geometry of nature, Freeman, New-York

[18] W. Werner (1995): On Brownian disconnection exponents, Bernoulli 1, 371-380. 
[19] W. Werner (1996): Bounds for disconnection exponents. Electronic Comm. Prob. 1, 19-28.

[20] W. Werner (1996): Asymptotic behaviour of disconnection and non-intersection exponents. Probab. Theor. Rel. Fields, to appear. 